\title{
Mu Heavy Chain Disease
}

National Cancer Institute

\section{Source}

National Cancer Institute. Mu Heavy Chain Disease. NCI Thesaurus. Code C3892.

A clonal disorder characterized by the secretion of a mu heavy chain that lacks a variable region. Most patients present with slowly progressive chronic lymphocytic leukemia (CLL).

In contrast to most cases of CLL, Mu heavy chain disease is associated with hepatosplenomeg aly and absence of lymphadenopathy. --2004 